Elsevier

BRD 50110

\title{
Trophic Capacity of Experimentally Lengthened Gustatory Axons
}

\author{
BRUCE OAKLEY, ELIZABETH KEPPEL and STEPHEN E. HUGHES \\ Department of Zoology, Neuroscience Laboratory Building, University of Michigan, Ann Arbor, MI 48109 (U.S.A.)
}

(Accepted May 29th, 1984)

Key words: axon - regeneration - taste - trophic - glossopharyngeal nerve - vagus nerve - gerbil

\begin{abstract}
Long nerve stumps will maintain end-organs for a longer period of time than short nerve stumps. However, longer maintenance might result not from a longer nerve stump per se but from uncontrolled factors such as less interference with the target's blood supply when transecting a nerve far from its target. To control for the site of nerve transection and to evaluate the trophic capacity of lengthened axons, we extended the gerbil's (Meriones unguiculatus) IXth nerve (glossopharyngeal) by splicing in a $5.0 \mathrm{~mm}$ segment of the superior laryngeal nerve. Thus, when assessing nerve stump-length dependency 3-5 months later, both the control regenerated nerve and the lengthened experimental nerve could be transected at the same site on the IXth nerve trunk. The peak magnitude of the summated taste discharges of the experimentally lengthened IXth nerve declined at a rate exactly predicted by an earlier study of nerve stump-length dependency. We conclude that maintenance of synaptic transmission depends upon taste axon stump-length and not upon factors uncontrolled in conventional experimental designs of stump-length dependency which transect nerves at different locations. Control regenerated and experimentally lengthened nerves maintained similar numbers of taste buds. Thus, neither the added burden of maintaining longer axons nor the presence of Schwann cells of the superior laryngeal nerve splice degraded the IXth nerve's trophic performance.
\end{abstract}

\section{INTRODUCTION}

Transection of a nerve trunk causes profound anterograde and retrograde changes. In the retrograde direction the neuron's soma may undergo chromatolysis while the synaptic boutons of other neurons which contact the soma may retract, thereby disabling the synaptic input to the injured neuron ${ }^{37}$. In the anterograde direction isolated mammalian axons are soon unable to activate their target synaptically and subsequently, after 1-3 days, are unable to propagate impulses $8,28,34$. Ultimately the distal portion of the transected nerve undergoes Wallerian degeneration.

Observations on the degeneration of isolated axons led Cajal to consider the cell body as the trophic center of the neuron ${ }^{33}$. In subsequent years a firm foundation for Cajal's formulation has emerged from extensive studies on axonal transport which have revealed a remarkable interchange between the soma and the axon terminals of materials concerned with maintenance of the cytoskeleton, metabolism and synaptic transmission. Thus, in many neurons the rapid anterograde transport of substances to the axonal terminals includes macromolecules essential for synaptic transmission 9,16 . In addition to maintaining nerve endings and synaptic transmission, neurons also trophically maintain certain kinds of targets including skeletal muscle, taste buds and the lateral geniculate nucleus (LGN). Clearly, maintenance of axonal endings, synaptic transmission and target cells must involve some processes in common although the term 'trophic' is often, but not invariably ${ }^{17}$, reserved for the last of these, namely, the maintenance of target cells.

The anterograde effects of axotomy upon transsynaptic signal transmission have been studied most extensively at neuromuscular synapses and LGN synapses. Synaptic transmission from a freshly transected optic nerve to LGN neurons lasts for at least 2-3 days before deteriorating 13,31 . It is only after the failure of synaptic transmission at optic nerve-LGN synapses that impulse propagation in the optic nerve fails and, ultimately, the LGN neurons atrophy.

Correspondence: B. Oakley, Neuroscience Laboratory Building, University of Michigan, Ann Arbor, MI 48109, U.S.A. 
Thus, a change in anterograde synaptic transmission is one of the earliest functional effects of denervation. However, within seconds after optic nerve section, the LGN cells become hyperexcitable, probably because of reduced interneuronal inhibition ${ }^{12}$. Thus, in central structures such as the LGN and spinal $\operatorname{cord}^{20}$, immediate physiological changes like disinhibition may mask acute deterioration of afferent synaptic transmission.

It is important to study a model system free of such neural network complexities because early changes in synaptic transmission may be part of the chain of events leading to end organ degeneration. Actual end organ degeneration measured days after nerve transection may be so indirect a consequence of lost neurotrophic influence that it is difficult from this delayed degenerative event to infer which specific properties are directly neurotrophically maintained.

Studies of the peripheral nervous system have more clearly revealed the rapid deterioration which occurs after nerve section. In muscle, evoked endplate potentials are reduced within $9 \mathrm{~h}$ and miniature endplate potentials decreased within $12 \mathrm{~h}$ after nerve section ${ }^{7.24}$. Early changes in the target muscle cells also occur; Albuquerque et al. ${ }^{2}$ reported reduced muscle membrane resistance $2-3 \mathrm{~h}$ after nerve section.

That the changes in denervated skeletal muscle reflect the loss of neurotrophic maintenance has been challenged by evidence favoring a predominant role for muscle activity in the maintenance of muscle $21.22,40.41$. For example, electrical activation of muscle can in large part replace the trophic influences of motor axons in establishing and maintaining muscle properties. However, some evidence suggests that muscle activity may not completely explain the neural maintenance of muscle properties. First, the restoration of muscle properties by electrical stimulation is incomplete ${ }^{41}$. Second, muscle degeneration can be induced by using colchicine to disrupt axonal transport while leaving muscle activity intact 3.5 .15 .38 . Similarly in the sense of taste, by controlling for the direct effects of colchicine upon the taste buds, a neurotrophic role of axonal transport has been indicated ${ }^{35}$. Third, many experiments indicate that the length of the peripheral nerve stump influences the rate of development of denervation-induced changes; deterioration of the end organ occurs more rapidly with short nerve stumps. A longer stump delays the appearance of physiological changes such as the decrease in resting membrane potential ${ }^{6}$, the appearance of extrajunctional acetylcholine sensitivity 23,39 and the decrease in miniature endplate potential24, and also delays biochemical changes in endplate acetylcholinesterase activity ${ }^{11}$. RNA synthesis ${ }^{32}$ and protein phosphorylation ${ }^{14}$.

Degeneration which is dependent on the length of the nerve stump might occur if shorter nerve stumps degenerate more rapidly ${ }^{24}$ and if the target is sensitive to the degeneration by-products ${ }^{40.41}$. To test this proposition isolated nerve segments or short lengths of thread were implanted directly onto the surface of a normally innervated muscle. 'Denervation' sensitivity to acetylcholine $(\mathrm{ACh})$ developed in the locally disturbed region of the muscle ${ }^{18}$. It is uncertain whether this effect was mediated by nerve degeneration by-products or by local damage to the muscle. Thus, it is possible that a denervated target degenerates more rapidly not because short stumps produce degeneration by-products more quickly nor because short nerve stumps provide less axonally transported trophic material but because operative trauma impairs the local blood supply, injures the nearby tissue or injures the target itself (vide infra).

The present study used mammalian taste buds as a model of trophic dependence. Taste buds are composed of clusters of slender, modified epithelial cells which synapse with fine branches of axons innervating the gustatory epithelium. Taste buds in several vertebrate groups have been shown to be trophically dependent upon their nerve supply. In the most sensitive mammalian preparations, transection of the gustatory nerves can cause degeneration of the taste buds within 3-7 days ${ }^{19.35}$. The reappearance of taste buds upon reinnervation by chemosensory axons confirms the trophic dependence of the taste buds upon their innervation (reviewed in ref. 25). Further support for neural maintenance is provided by studies which have shown that the maintenance of both taste responses ${ }^{27}$ and taste buds ${ }^{36}$ depends upon the length of the nerve stump.

We chose to examine acute and chronic anterograde changes in the peripheral taste system in response to transection of a nerve trunk. The experimental designs of previous studies on nerve stumplength dependency of taste buds and muscle have not 
included controls for the site of nerve transection. While obviously producing shorter axon stumps, the operative procedure to expose and transect the nerve near to the target may in particular interrupt axons joining the nerve trunk distally through small anastomoses or may cause local trauma and disruption of the target's blood supply. In addition to controlling for such potential problems, we were also interested in establishing whether the trophic capacity of gustatory axons might be weakened as the result of either nerve elongation or association with new Schwann cells from a nerve splice. Hence, we extended the IXth nerve of gerbils by causing the axons to regenerate through a segment of the vagus nerve. The trophic capacity of these experimentally lengthened IXth nerves was compared with that of control regenerated IXth nerves which lacked the nerve splice, but were transected at the same locus as the experimental nerve. Thus, in addition to addressing general concerns about the conventional design of nerve stump-length dependency studies, we reasoned that proportionally longer maintenance of taste responses by experimentally lengthened nerves would argue for flexible adjustment of functional maintenance by lengthened axons, and argue against a requirement for a specific type of Schwann cell along the entire route of the nerve.

The taste system is notable as the only trophic system under active investigation in which the axon terminal is postsynaptic. It avoids problems encountered in the analysis of skeletal muscle because with taste buds efferent impulse activity appears to be irrelevant to neurotrophic maintenance ${ }^{4,11}$.

\section{MATERIALS AND METHODS}

Male and female Mongolian gerbils (Meriones unguiculatus), aged 4-12 months, were anesthetized with ketamine hydrochloride $(325 \mathrm{mg} / \mathrm{kg}$ body weight, i.m.). In 5 control gerbils the right IXth nerve was transected about $4 \mathrm{~mm}$ distal to the petrosal ganglion and the ends reunited with a suture. Ethilon 11-0 black monofilament nylon (Ethicon, Inc.) was used for all sutures in this study. In 10 experimental gerbils the right IXth nerve was transected in an identical manner but was extended by a $4.5-5.0 \mathrm{~mm}$ segment of the superior laryngeal nerve branch of the left Xth nerve (SLN, vagus) spliced be-

\section{EXPERIMENTAL}

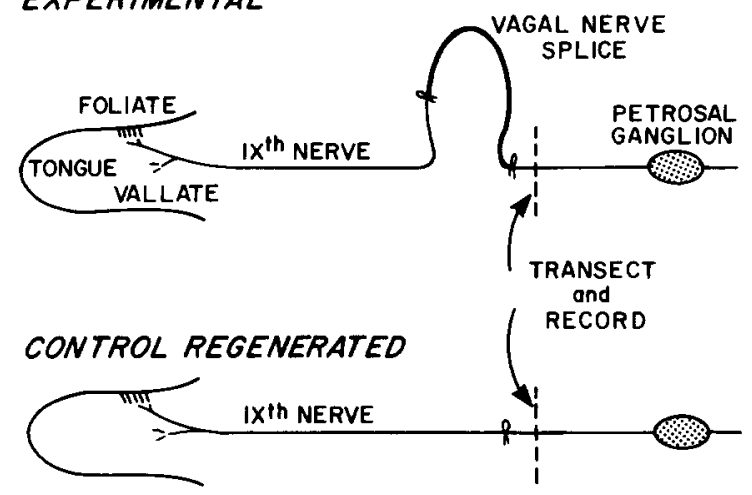

Fig. 1. Schematic drawing showing the method of lengthening IXth nerve axons by having them regenerate through an isolated segment of the autogenous superior laryngeal nerve (vagus) which was approximately equal to the IXth nerve in diameter. In the control animals, the IXth nerve was transected and sutured together. Three to 5 months later, both the experimental and control regenerated IXth nerves were transected at the same site for electrophysiological evaluation of the nerve stump-length dependency of the taste discharges recorded from the proximal end of the distal nerve stump.

tween the distal and the proximal cut ends of the IXth nerve (Fig. 1). In the control animals the identical portion of the left SLN was excised. To reduce the possibility of regenerating IXth nerve axons escaping via the stumps of lateral branches of the nerve splice, the SLN was spliced in reversed orientation such that one suture united the proximal end of the IXth nerve with the distal stump of the SLN and another suture united the proximal end of the SLN with the distal stump of the IXth nerve. Thus, severed axons in the proximal portion of the experimental IXth nerve regenerated to the tongue through an approximately equal-diameter SLN conduit containing Schwann cells and degenerated axon fragments. If the regenerated IXth nerve axons had a maintenance action that was proportional to their added length, the $5 \mathrm{~mm}$ splice length would clearly be long enough to prolong the taste response.

After allowing a minimum of 3 months for nerve regeneration, the gerbils were anesthetized with ketamine followed 30 min later by sodium pentobarbital (12 $\mathrm{mg} / \mathrm{kg}$, i.p.) with supplementary doses of sodium pentobarbital $(8.5 \mathrm{mg} / \mathrm{kg})$ as needed. A tracheal cannula was inserted, and the esophagus and upper portion of the trachea ligated to prevent seepage of taste solutions. The regenerated IXth nerve was exposed, transected near the proximal suture and lifted onto a 
pair of $100 \mu \mathrm{m}$ diameter nichrome wire recording electrodes. For all control and experimental animals the IXth nerve was transected at the same location (Fig. 1). The multi-unit impulse discharges were recorded from the transected nerve, and were amplified and displayed on an oscilloscope. The recorded impulses were stored on magnetic tape and were electronically summated (Grass 7P3A, $0.5 \mathrm{~s}$ time constant) for on-line registration with a polygraph. The response measure was the peak amplitude of the summated nerve discharge in excess of the pre-stimulus baseline level of activity. With the gravity flow system, each taste solution was applied to the tongue for $20 \mathrm{~s}$ followed by $220 \mathrm{~s}$ of distilled water rinse. The taste solutions, $0.3 \mathrm{M} \mathrm{NH}_{4} \mathrm{Cl}$ and $0.5 \mathrm{M}$ sucrose, were made from reagent grade chemicals dissolved in glass-distilled water and were used in alternation with distilled water rinse. The temperatures of the gerbil's body and of the taste solutions and rinse water were maintained at $35-37^{\circ} \mathrm{C}$ as monitored by thermocouples. A further description of recording methods may be found in ref. 28 .

To determine the final length of the SLN splice after 3-5 months of recovery, the splice length was measured at the close of electrophysiological recording as the distance between the two sutures. The total length of the control transected IXth nerve was obtained from earlier measurements ${ }^{27}$. After the recording session, the tongue was removed and immersed in formalin-sucrose- $\mathrm{NH}_{4} \mathrm{OH}$ (10\%:15\%: $1 \%)$. Each tongue was embedded in paraffin and sectioned serially at $10 \mu \mathrm{m}$. The sections were stained in Heidenhain's iron hematoxylin to identify foliate and vallate taste buds by the presence of a darkened taste pore region. Vallate taste buds were counted in 7 normal gerbils.

\section{RESULTS}

The extended IXth nerves and control regenerated IXth nerves responded similarly to the taste solutions. Apart from a lower level of spontaneous activity and a reduced signal-to-noise ratio, the responses to $\mathrm{NH}_{4} \mathrm{Cl}$ and sucrose in the regenerated nerves immediately after nerve transection were as predicted from a study of normal IXth nerves ${ }^{29}$. Subsequent responses had progressively lower amplitudes, since transection of the gerbil's IXth nerve results in a

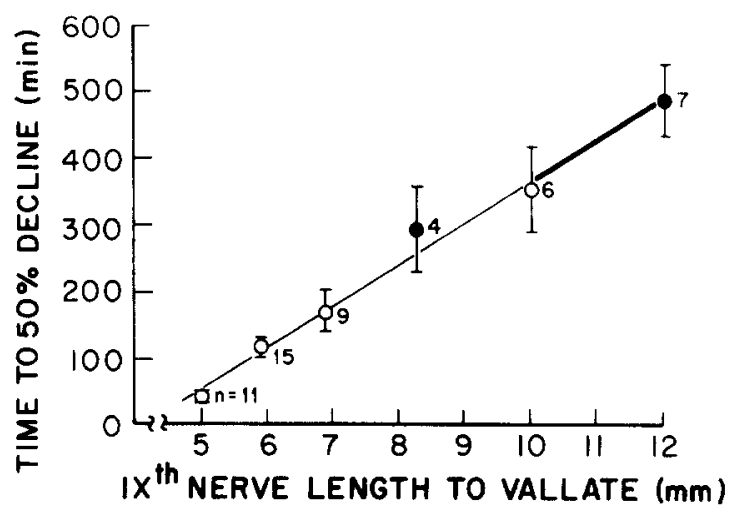

Fig. 2. Following transection of the regenerated nerves, the latency to a $50 \%$ decline in taste impulse discharges is a function of the length of the IXth nerve stump remaining attached to the tongue. Open circles: normal IXth nerves with stump-lengths set by locus of transection ${ }^{27}$. Filled circle, $n=4$ : control regenerated IXth nerves. Filled circle, $\mathbf{n}=7$ : experimental regenerated IXth nerves extended by regrowth through a vagal nerve splice. The thick line segment is a linear continuation of the line fitted to the open circles by the least squares method. Stimulus: $0.3 \mathrm{M} \mathrm{NH}_{4} \mathrm{Cl}, \mathrm{n}=52$ IXth nerves (41 normal nerves and 11 regenerated). Error bars $= \pm 1$ S.E.

rapid loss of taste responses several hours before impulse propagation mechanisms are $\operatorname{los}^{28}$. Earlier studies of this phenomenon had shown that the latency of the response decline was proportional to the length of the nerve stump remaining attached to the tongue ${ }^{27}$. The open circles in Fig. 2 were obtained with this conventional experimental design of nerve stump-length dependency ${ }^{27}$. They indicate the time required for taste response magnitudes to fall to $50 \%$ of their initial value in gerbil IXth nerves transected at the indicated distances from the vallate papilla. The data show a linear nerve stump-length dependency.

In the present study of extended IXth nerves, recording difficulties prevented us from obtaining taste responses from the initial preparations. Taste responses were obtained from 4 of 5 control and 7 of 10 experimental animals. The control regenerated IXth nerves (the filled circle at $8.3 \mathrm{~mm}$, Fig. 2) maintained taste responses for a time period which agreed with the previously determined nerve stump-length relationship. The experimentally extended IXth nerves (the filled circle at $12 \mathrm{~mm}$ ) maintained taste responses for the longest period of time, in strict proportion to their longer nerve stump-length. Hence, 
the taste responses of the experimental animals declined to $50 \%$ of their initial magnitude after a delay significantly longer than in the control animals (mean $=485$ vs $294 \mathrm{~min}, P=0.036$ Mann-Whitney $\mathrm{U}$ test).

We counted foliate and vallate taste buds in normal, control and experimental gerbils. Because the single vallate papilla lies on the midline of the tongue, many of its taste buds received innervation from both the intact left and regenerated right IXth nerves. However, the foliate taste buds, which lie at the lateral margins of the posterior portion of the tongue, are unilaterally innervated by the IXth nerve with a small, stable contribution from the ipsilateral chorda tympani nerve (Hosley and Oakley, unpublished observations). Hence, the foliate taste buds ( $\overline{\mathrm{X}}$ $=132$ ) are the more appropriate assay system for the trophic consequences of unilateral manipulations of the IXth nerve. Although the $17 \%$ reduction of foliate taste buds in the control and the $21 \%$ reduction in the experimental animals imply incomplete nerve regeneration, these percentage changes did not differ statistically from normal animals $(P>0.05$, Mann-Whitney U-test). Nor were there significant differences between experimental and control animals in the number of: foliate taste buds on the operated side $(P>0.1)$, vallate taste buds $(P>0.05)$, or vallate and foliate taste buds combined $(P>0.1$,

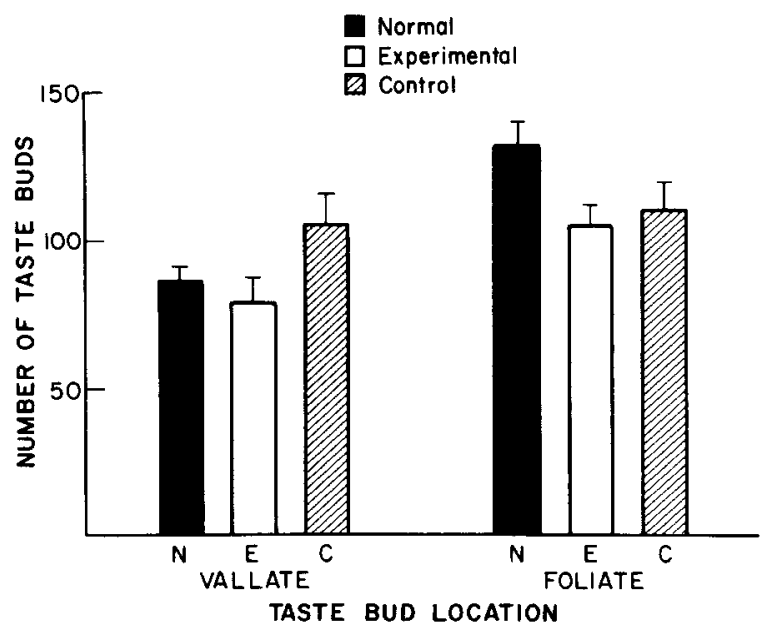

Fig. 3. Number of taste buds in vallate and foliate papillae innervated by normal $(N)$, control regenerated $(C, n=5)$, and experimentally lengthened $(E, n=10)$ IXth nerves. Normal foliate counts are from the contralateral foliate papillae $(n=15)$, and normal vallate counts are from normal gerbil tongues $(\mathbf{n}=$ 7). Error bars $= \pm 1$ S.E.
Fig. 3). Vallate and foliate taste buds were also just as numerous in those animals from which taste responses were not obtained due to recording difficulties. In summary, the number of foliate and vallate taste buds provided anatomical evidence that the chronic trophic capacity of the control and experimental regenerated nerves was not diminished.

The electrophysiological tests and taste bud counts were carried out after mean recovery periods of 128 days (range $=86$ to 168 ) for the experimental animals and 135 days (range $=88$ to 152 ) for the controls. Neither the time to $50 \%$ response decline nor the number of regenerated taste buds in the foliate papillae was related to the duration of the recovery period. This suggests that after a recovery period of 3 months or longer, we were examining stable properties of the system.

\section{DISCUSSION}

Taste bud morphology reflects the long-term neurotrophic maintenance of the end organ, while taste nerve impulse discharges reflect the shorter-term physiological maintenance of the taste bud system, including synaptic transmission. We used impulse discharges of the whole IXth nerve as a convenient, acute bioassay for physiological performance. In trying to understand the nature of trophic dependence in the taste system it is important to investigate the earliest impairments.

In the present study the duration of maintenance of taste discharges in both control regenerated and experimentally extended IXth nerves agreed with the previously determined nerve stump-length relationship ${ }^{27}$. Both the experimental and control nerves were transected at the same locus. Thus, the longer maintenance of the taste responses observed for the extended IXth nerves cannot be attributed either (i) to a lesser disruption of the blood supply to the taste buds or nerve trunk, (ii) to intact nerve branches which join the trunk distally or (iii) to reduced salivary secretion into the clefts of the gustatory papillae, thereby allowing better stimulation of the taste buds. Rather, it is the experimental variable of the added axon length which accounts for a prolonged response in the transected experimental nerve. Lengthened axons maintained taste discharges longer. 
Aguayo et al." have shown by autoradiography that sural nerve axons regenerating through a $5 \mathrm{~mm}$ segment of the cervical sympathetic trunk become myelinated by the Schwann cells surviving in the sympathetic trunk. Thus, it is likely that the IXth nerve axons were myelinated by Schwann cells of the $5 \mathrm{~mm}$ SLN splice which we used. Nonetheless, such associations of a portion of the extended axons with SLN Schwann cells did not impair the trophic capacity of taste axons in supporting taste buds. Thus, there is no evidence to suggest that the supply of trophic materials is normally derived from or stimulated by specific Schwann cells associated with gustatory axons.

If one judges from the similar number of foliate taste buds reformed and maintained by control and by lengthened nerves, the necessity to maintain an added $40 \%$ of axon length did not impair the trophic capacity of the lengthened axons. Maintenance of the extended axons themselves may be aided by feedback regulation of the supply of essential materials from the cell body.

It is the axon terminals of transected nerves which fail to support taste buds. That is, the degeneration of taste buds must be secondary to some earlier alteration of the postsynaptic axonal terminals. Presumably, the taste bud is normally maintained either by axonal release of a diffusible trophic chemical agent(s) or by an interaction of membrane elements of the axon and epithelial cell. Disruption in chemical release or membrane interaction heralds the onset of degenerative changes in the end organ. The soma of taste neurons has the dual role of maintaining not only the axon terminal but also the end organ. In current usage the word trophic (Greek: nourishing) refers to neuronal maintenance of the normal morphological, physiological and biochemical features of another cell or tissue. But the causal linkages make it necessary to consider the neuron's trophic role in maintaining its own axon in the classic sense of $\mathrm{Ca}$ -

\section{REFERENCES}

1 Aguayo, A. J., Charron, L. and Bray, G. M., Potential of Schwann cells from unmyelinated nerves to produce myelin: a quantitative ultrastructural and radiographic study, $J$. Neurocytol., 5 (1976) 565-573.

2 Albuquerque, E. X., Schuh, F. T. and Kauffman, F. C., Early membrane depolarization of the fast mammalian jal ${ }^{17.33}$. Although the trophic maintenance of end organs can be highly dramatic in selected instances, the virtually ubiquitous nutritive maintainance of axon terminals by the neuron's soma indicates the fundamental biological importance of that process.

It is likely that materials carried from the cell body by axonal transport sustain the structure and metabolism of both the axon terminals and end organs. Materials, like cytoskeletal elements, which are transported at slow rates (1-4 mm/day) are probably not crucial for the immediate maintenance of the taste response or the taste bud which depend upon faster transport rates $^{27.35}$. Since oxygen-dependent axonal transport continues in isolated nerves ${ }^{26.30}$, the rapid failure of the taste response would not appear to result from a cessation of oxidative metabolism in axon terminals. Nor would the axonal delivery of neurotransmitter seem to be relevant since the terminals of taste axons are postsynaptic to the receptor cells which are believed to contain the chemical transmitter.

In conclusion, the present experimental design controlled for artifacts that might arise from varying the site of nerve transection in studies of nerve stump-length dependency. This report on regenerated taste nerves indicates that the maintenance of taste responses is proportional to the distal nerve stump-length even in experimentally lengthened nerves. Neither association with Schwann cells of the $X$ th nerve nor lengthening of the axons degraded the experimental IXth nerve's trophic capacity to maintain typical numbers of taste buds or to maintain taste responses after nerve transection.

\section{ACKNOWLEDGEMENTS}

We are grateful to Linda Morton for valued assistance. Supported in part by NIH Grant NS-07072.

muscle after denervation, Pflügers Arch., 328 (1971) $36-50$.

3 Albuquerque, E. X., Warnick, J. E., Tasse, J. R. and Sansone, F. M., Effects of vinblastine and colchicine on neural regulation of the fast and slow skeletal muscles of the rat. Exp. Neurol., 37 (1972) 607-634.

4 Berland, D. W., Chu, J. S., Hosley, M. A., Jones, L. B., Kaliszewski, J, M. . Lawler, W. C. and Oakley, B., New ap- 
proaches to the problem of the trophic function of neurons. In J. Le Magnen and P. MacLeod (Eds.), Olfaction and Taste VI, Information Retrieval, London, 1977, pp. 217-224.

5 Cangiano, A., Acetylcholine supersensitivity: the role of neurotrophic factors, Brain Res., 58 (1973) 255-259.

6 Card, D. J., Denervation: sequence of neuromuscular degenerative changes in rats and the effect of stimulation, Exp. Neurol., 54 (1977) 251-265.

7 Card. D. J., Physiological alterations of rat extensor digitorum longus motor nerve terminals as a result of surgical denervation, Exp. Neurol., 54 (1977) 478-488.

8 Causey, G. and Stratmann, C. J., The spread of failure of conduction in degenerating mammalian nerve, $J$. Physiol. (Lond.), 121 (1953) 215-223.

9 Dahlström, A., Axoplasmic transport (with particular respect to adrenergic neurons), Phil. Trans. B, 261 (1971) 315 -358 .

10 Davey, B. and Younkin, S. G., Effect of nerve stump length on cholinesterase in denervated rat diaphragm, Exp. Neurol., 59 (1978) 168-175.

11 Donoso, A. and Zapata, P., Effects of denervation and decentralization upon taste buds, Experientia (Basel), 32 (1976) 591-592.

12 Eysel, U. T. and Grüsser, O.-J., Increased transneuronal excitation of the cat lateral geniculate nucleus after acute deafferentation, Brain Res., 158 (1978) 107-128.

13 Eysel, U. T., Grüsser, O.-J. and Pecci Saavedra, J., Signal transmission through degenerating synapses in the lateral geniculate body of the cat, Brain Res., 76 (1974) 49-70.

14 Held, I. R., Squinto, S. P. and McLane, J. A., Neurotrophic regulation of the phosphorylation of a soluble cytosolic protein in skeletal muscle, J. Neurosci., 3 (1983) 2054-2063.

15 Hoffmann, W. W. and Thesleff, S., Studies on the trophic influence of nerve of skeletal muscle, Europ. J. Pharmacol., 20 (1972) 256-260.

16 Holmgren, E., Karlsson, J.-O. and Sjöstrand, J., Changes in synaptic function induced by blockage of axonal transport in the rabbit optic pathway, Brain Res., 157 (1978) $267-276$.

17 Hopkins, W. G. and Brown, M. C., Development of Nerve Cells and their Connections, Cambridge University Press, Cambridge, 1984, p. 104.

18 Jones, R. and Vrbova, G., Two factors responsible for the development of denervation hypersensitivity, $J$. Physiol. (Lond.), 236 (1974) 517-538.

19 Kennedy, J. G., The effects of transection of the glossopharyngeal nerve on the taste buds of the circumvallate papilla of the rat, Arch. Oral Biol., 17 (1972) 1197-1207.

20 Kostyuk, P. G., Functional changes during degeneration of central synapses. In E. Gutman and P. Hnik (Eds.), The Effect of Use and Disuse on Neuromuscular Function, Elsevier, Amsterdam, 1963, pp. 291-304.

21 Lømo, T. and Rosenthal, J., Control of ACh sensitivity by muscle activity in the rat, J. Physiol. (Lond.), 221 (1972) 493-513.

22 Lømo, T. and Westgaard, R. H., Further studies on the control of ACh sensitivity by muscle activity in the rat, $J$. Physiol. (Lond.), 252 (1975) 603-626.
23 Luco, J. V. and Eyzaguirre, C., Fibrillation and hypersensitivity of $\mathrm{ACh}$ in denervated muscle: effect of length of degenerating nerve fibers, $J$. Neurophysiol., 18 (1955) 65-73.

24 Miledi, R. and Slater, C. R., On the degeneration of rat neuromuscular junctions after nerve section, J. Physiol. (Lond.), 207 (1970) 507-528.

25 Oakley, B., Trophic competence in mammalian gustation. In D. Pfaff (Ed.), Olfactory and Gustatory Influences on the Central Nervous System, The Rockefeller Univ. Press, in press.

26 Oakley, B., Chu, J. S. and Jones, L. B., Axonal transport maintains taste responses, Brain Res., 221 (1981) 289-298.

27 Oakley, B., Jones, L. B. and Hosley, M. A., The effect of nerve stump length upon mammalian taste responses, Brain Res., 194 (1980) 213-218.

28 Oakley, B., Jones, L. B. and Hosley, M. A., Decline of IXth nerve taste responses following nerve transection, Chem. Senses Flav., 4 (1979) 287-229

29 Oakley, B., Jones, L. B. and Kaliszewski, J. M., Taste responses of the gerbil IXth nerve, Chem. Senses Flav., 4 (1979) 79-87.

30 Ochs, S., Local supply of energy to the fast axoplasmic transport mechanism, Proc. nat. Acad. Sci. U.S.A., 68 (1971) 1279-1282.

31 Pecci Saavedra, J., Vaccarezza, O. L., Reader, T. A. and Pasqualini, E., Synaptic transmission in the degenerating lateral geniculate nucleus: an ultrastructural and electrophysiological study, Exp. Neurol., 26 (1970) 607-620.

32 Politoff, A. L. and Blitz, A. L., Neurotrophic control of RNA synthesis in amphibian striated muscles, Brain Res., 151 (1978) 561-570.

33 Ramon y Cajal, S., Textura del Sistema Nervioso del Hombre y de los Vertebrados, Vol. I, N. Moya, Madrid, 1899 , p. 30.

34 Rosenblueth, A. and Del Pozo, E. C., The centrifugal course of Wallerian degeneration, Amer. J. Physiol., 139 (1943) 247-254.

35 Sloan, H. E., Hughes, S. E. and Oakley, B., Chronic impairment of axonal transport eliminates taste responses and taste buds, J. Neurosci., 3 (1983) 117-123.

36 State, F. A. and Dessouky, H. I., Effect of length of the distal stump of transected nerve upon the rate of degeneration of taste buds, Acta Anat. (Basel), 98 (1977) 353-360.

37 Sumner, B. E. H. and Sutherland, F., Quantitative electron microscopy on the injured hypoglossal nucleus in the rat, $J$. Neurocytol., 2 (1973) 315-328.

38 Tiedt, T. N., Lewis Wisler, P. and Younkin, S. G., Neurotrophic regulation of resting membrane potential and acetylcholine sensitivity in rat extensor digitorum longus muscle, Exp. Neurol., 57 (1977) 766-791.

39 Uchitel, O. and Robbins, N., On the appearance of acetylcholine receptors in denervated rat diaphragm, and its dependence on nerve stump length, Brain Res., 153 (1978) 539-548.

40 Vrbova, G., Control of chemosensitivity at the neuromuscular junction. In R. Eigenmann (Ed.), Proc. 4th Int. Cong. Pharmacol., Vol. III, Schwabe and Co., Basel, 1970, pp. 158-169.

41 Vrbova, G., Gordon, T, and Jones, R., Nerve-Muscle Interaction, John Wiley, New York, 1978, 233 pp. 